\title{
Sexually dimorphic mechanosensory neurons regulate copulation duration and persistence in male Drosophila
}

\section{Shreyas Jois}

University of Saskatchewan

Yick-Bun Chan

Harvard Medical School

Maria Paz Fernandez

Barnard College

Narsimha Pujari

University of Saskatchewan

Lea Joline Janz

University of Saskatchewan

Adelaine Kwung-Wai Leung ( $\square$ adelaine.leung@usask.ca )

University of Saskatchewan

\section{Research Article}

Keywords: peripheral sensory neurons, Drosophila, fru/dsx

Posted Date: November 15th, 2021

DOI: https://doi.org/10.21203/rs.3.rs-1083236/v1

License: (c) (i) This work is licensed under a Creative Commons Attribution 4.0 International License. Read Full License 


\section{Abstract}

Peripheral sensory neurons are the gateway to the environment across species. In Drosophila, olfactory and gustatory senses are required to initiate courtship, as well as for the escalation of courtship patterns that lead to copulation. To be successful, copulation must last long enough to ensure the transfer of sperm and seminal fluid that ultimately leads to fertilization. The fly genitalia contain sex-specific bristle hairs innervated by mechanosensory neurons. To date, the role of the sensory information collected by these peripheral neurons in male copulatory behavior is unknown. Here, we employed genetic manipulations that allow driving gene expression in the male genitalia as a tool to uncover the role of these genitalia specific neurons in copulation. We found that the sensory information received by the mechanosensory neurons (MSNs) at the male genitalia plays a key role in copulation duration. We confirmed that these MSNs are cholinergic and co-express both fru and $d s x$. Moreover, our results show that the function of these $f r u / d s x$ cholinergic MSNs is required for copulation persistence, which ensures copulation is undisrupted in the presence of environmental stress before sperm transfer is complete.

\section{Introduction}

An animal's decision to perform a certain behavior requires the integration of external stimuli with homeostatic regulation. The nervous system collects environmental sensory information and integrates it with the internal status of the animal to generate motor signals that elicit an appropriate behavioral response. Drosophila male courtship is an excellent model to investigate how complex behavior is coordinated by the nervous system. The development of the courtship neural circuit is largely controlled by two sex determination genes, fruitless (fru) and doublesex $(d s x)^{1-3}$. The 2000 fru-expressing neurons, comprising of sensory, integration, and motor neurons, control most aspects of male courtship behavior $^{4-9}$. However, the complete courtship neural circuit also requires $d s x$ expressing neurons, a subset of which co-expresses $f u^{10-14}$.

Successful courtship requires the processing of sensory information received by peripheral sensory neurons ${ }^{15}$. In the antenna, fru olfactory receptor neurons (ORNs) receive and transmit volatile olfactory signals to the antennal lobe in the brain; fru ORNs expressing the receptor Or67d respond to the male pheromone cVA which inhibits mating behavior in males but promotes mating behaviors in females ${ }^{16}$. Gustatory receptor neurons (GRNs) in the front legs receive and transmit non-volatile gustatory signals to the CNS. For example, fru GRNs expressing the ion channels ppk23 and ppk29 are required to detect inhibitory signals on males and excitatory signals on females during courtship ${ }^{17}$. Dsx neurons expressing the receptor Gr68a respond to the anti-aphrodisiac pheromone $\mathrm{CH} 503$ and inhibit male courtship ${ }^{18}$. Acoustic information from the movement of the female detected by the Johnston's organ neurons (JONs) helps to promote courtship initiation in males ${ }^{19}$. Finally, the visual contribution to the initiation and maintenance of courtship has also been shown in a treadmill-based courtship assay ${ }^{20}$. 
When the female is receptive to male courtship, she will slow down and spread her wings, allowing the male to mount. The male initiates copulation by bending his abdomen forward to attach the genitalia. The male terminalia is divided in two regions: the periphallic structures and the intromittent organ. The periphallic structures form weaker connections to the intromittent organ and can be easily separated by dissection. The periphalilic structures are subdivided into four regions: the epandrial ventral lobe (formerly called: lateral plate) (EVL), the surstylus (formerly called: clasper) (SUR), the epandrial posterior lobe (formerly called posterior lobe) (EPL), and the cercus (formerly called: anal plate) (CER) ${ }^{21}$. Genital coupling, as revealed by a high-resolution electron microscopic time sequence analysis, involves the active movement of these periphalic structures ${ }^{22}$. The surstylus bends medially and is hidden from view. Within 10 minutes of copulation, the cercus aligns with the female oviscape and achieves genital coupling. Each periphallic region contains an array of stereotypic, species and sex-specific bristles, and each bristle is innervated by a bipolar mechanosensory neuron ${ }^{23}$. The neural implication of this sensory information collected during copulation is unclear.

Copulation must last long enough to ensure successful sperm transfer, which does not occur until five minutes after copulation starts ${ }^{24}$. The regulation of copulation time in Drosophila is a complicated process that does not simply depend on the volume or the transfer of sperm and seminal fluid, since mutants defective in their synthesis still have normal copulation duration ${ }^{24}$. Both neuronal and nonneuronal factors can influence mating time. Neuronal regulators include a small cohort of fru neurons that co-express the transcription factor engrai ${ }^{25}$ and a subset of 4-5 fru neurons that innervate the male reproductive tissues ${ }^{26,27}$. The latter neurons control copulation duration in response to sperm transfer ${ }^{26}$. Non-neuronal factors include mutations in the circadian clock gene period, which exhibit longer copulation duration ${ }^{28}$, and environmental factors such as the gut microbiome ${ }^{29}$ and environmental stressors ${ }^{24}$. Copulation persistence describes the maintenance of copulation in the presence of stressful stimuli, which peaks within the first 5 minutes of copulation and decreases over time ${ }^{24}$. This is an important cost-benefit analysis that ensures species survival. The neural circuit for this cost-benefit analysis includes $8 d s x / G A B A$ neurons in the ventral cord that decrease copulation persistence. With opposite action, ventral cord DA neurons increase copulation persistence ${ }^{24}$. However, the neural input required to initiate copulation persistence is unknown. In this study, we show that sensory information received by the mechanosensory neurons (MSNs) at the male genitalia is a novel neuronal regulator of copulation duration and is critical for the maintenance of copulation in the presence of stressful stimuli before sperm transfer is complete.

\section{Results}

\section{Discovery of fruitless (fru) neurons that regulate copulation duration}


To uncover the neuronal regulators of copulation duration, we utilized an intersectional genetic approach, involving both the GAL4/UAS ${ }^{30}$ and FLP/FRT ${ }^{7}$ expression systems. The details of the intersectional genetic system and how we generated a FLP enhancer trap screen had been described elsewhere $27,31,32$. Briefly, the target gene is downstream of a DNA sequence that contains the binding site (UAS) for the transcription factor GAL4, followed by a DNA sequence that contains a stop codon flanked by the FLP recombinase recognition site FRT (UAS-FRT-stop-FRT-target gene). A cell must possess an active promoter for FLP (to remove the stop codon by recombination) and an active promoter for GAL4 (to bind to UAS) to activate expression of the target gene. As an additional tool to restrict gene expression, tsh ${ }^{\mathrm{GAL} 80}$ is used to inhibit GAL4 expression specifically in the ventral cord. From here forward, the genetic nomenclature will list all transgenes that are responsible to drive expression of the target gene (e.g. " $\mathrm{X}{ }^{\mathrm{GAL}}{ }_{\text {,FLP }}{ }^{\#}>\operatorname{target}$ gene" denotes a fly that carries a GAL4 driver controlled by the promoter of X, and the FLP line \# from the enhancer trap screen, expressing the target gene). For the relevant neuronal populations, we expressed either GFP (UAS-FRT-stop-FRT-GFP) to visualize the morphology or the neuronal silencer tetanus toxin (UAS-FRT-stop-FRT-TNT) to block neuronal activity. Previously, we identified a cluster of $\sim 5 \mathrm{fru}$ 5HT/DA neurons (fru-sAbg-1) in the male abdominal ganglion that innervate various male reproductive tissues and regulates copulation duration ${ }^{27}$. In that study, we observed a reduction in copulation duration when we combined FLP335 with either fru-GAL, that targets most fruitless neurons

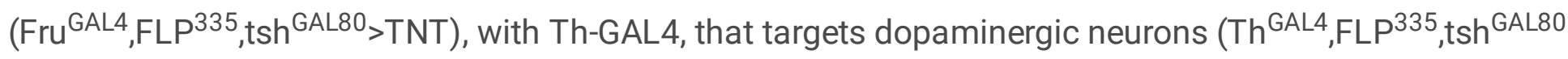
$>\mathrm{TNT}$ ), or with $5 \mathrm{HT}-\mathrm{GAL} 4$, that targets serotonergic neurons $\left(5 \mathrm{HT}^{\mathrm{GAL}}{ }^{\mathrm{G}}, \mathrm{FLP}^{335}, \mathrm{tsh}^{\mathrm{GAL} 80}>\mathrm{TNT}\right)^{27}$. However, neither silencing the subsets of dopaminergic nor serotonergic neurons can recapitulate the copulation phenotype observed when we targeted the broader fruitless circuit. The result suggested that additional fru neurons contributed to the copulation duration phenotype ${ }^{27}$. Indeed, the expression pattern for $\mathrm{Fru}^{\mathrm{Gal} 4}{ }^{,} \mathrm{FLP}^{335}{ }^{\text {,tsh }}{ }^{\mathrm{GAL} 80}>\mathrm{GFP}$ males extended beyond the accessory glands and seminal vesicles all the way to the genitalia (Figure 1A). Upon further examination, we were able to trace GFP expression to the male genitalia.

To further confirm the genitalia neurons are implicated in copulation duration control, we screened our enhancer trap FLP library (ref?) to search for other FLP lines that affected copulatory behaviors. We identified another FLP line that showed expression at the genitalia and also reduced copulation duration when it is combined with fru-GAL4 to express TNT in males (Fru ${ }^{G A L 4}, \mathrm{FLP}^{386}, \mathrm{tsh}^{\mathrm{GAL} 80}>\mathrm{TNT}$ ) (Figure 1D). The experimental males ( $\mathrm{Fru}^{\mathrm{Gal} 4}, \mathrm{FLP}^{386}{ }^{\mathrm{tsh}}{ }^{\mathrm{GAL} 80}>\mathrm{GFP}$ ) showed similar expression in fru ORNs, JONs, and GRNs compared to Fru ${ }^{\mathrm{GAL} 4}{ }_{\text {,FLP }}{ }^{335}$,tsh $^{\mathrm{GAL} 80}>\mathrm{GFP}$ males (Figure 1B). However, the prominent fru-sAbg-1 neurons in the abdominal ganglion that innervate the male reproductive tissue were noticeably missing, leaving only the sexually dimorphic arborizations (ANN1) in the abdominal ganglion originating from the MSNs in the genitalia. The expression pattern in the peripheral tissues that extend out from the ventral cord of $\mathrm{Fru}^{\mathrm{GAL} 4}{ }^{\text {,FLP }}{ }^{386}$,tsh ${ }^{\mathrm{GAL} 80}>\mathrm{GFP}$ males was highly restricted, showing GFP expression only in the male genitalia (Figure 1B). Although we still observed expression for fru GRNs, it is less consistent compared to $\mathrm{Fru}^{\mathrm{GAL} 4}, \mathrm{FLP}^{335}$,tsh ${ }^{\mathrm{GAL} 80}>\mathrm{GFP}$. In summary, three common fru neurons are consistently labeled by both FLP lines: 1) the sexually dimorphic projections in the glomeruli from fru ORNs (DA1, 
VA1v, VL2a), 2) projections in the antennal mechanosensory motor complex (AMMC) from fru JONs, and 3) projections in the abdominal ganglion (ANN1) from fru MSNs in the genitalia (Figure 1C, Table 1). The copulation duration of $\mathrm{Fru}^{\mathrm{GAL}}{ }^{\mathrm{G}}, \mathrm{FLP}^{386}$, tsh $^{\mathrm{GAL} 80}>\mathrm{TNT}$ was shortened by $26 \%$ compared to the TNT inactive control (Figure 1D). This difference is comparable to that observed in Fru $^{\text {GAL4 }}{ }^{\text {,FLP }}{ }^{335}{ }_{\text {,tsh }}{ }^{\text {GAL80 }}>$ TNT males. Of the three groups of fru neurons targeted by both FLP lines, we hypothesized that ANN1 from fru MSNs in the genitalia were the most likely to contribute to the copulation duration phenotype since we showed previously that silencing fru ORNs and GRNs did not affect copulation duration ${ }^{27}$. Recently, it was discovered that females sing in copula and that this song influences the reproductive success of the male ${ }^{33}$. However, the study showed that copulation duration was unaffected by the absence of female singing ${ }^{33}$.

\section{Characterization of fru genitalia mechanosensory neurons (MSNs)}

We further characterized the expression pattern in the genitalia terminals using various genetic combinations. The complete fru circuit (fru>GFP) in males show consistent GFP expression in a subset of mechanosensory neurons in the epandrial ventral lobe $(10 \pm 4, \sim 50 \%$ of EVL bristles), the surstylus ( $16 \pm 2$, $\sim 64 \%$ of SUR bristles), and the cercus $(20 \pm 2, \sim 61 \%$ of CER bristles) (Figure $2 B$, Table 2 ). Adding FLP 335 or FLP $^{386}$ eliminated GFP expression in the epandrial ventral lobe and restricted expression in fru MSNs at the surstylus and the cercus (Figure $2 \mathrm{C}$, Table 2). Amongst these two lines, Fru ${ }^{\mathrm{GAL}}{ }^{,} \mathrm{FLP}^{386}, \mathrm{tsh}^{\mathrm{GAL} 80}>\mathrm{GFP}$ revealed a more restrictive expression pattern with $8 \pm 3$ fru neurons at the surstylus and $4 \pm 2$ at the cercus (Table 2). As Fru ${ }^{\text {GAL4 }}$,FLP ${ }^{386}$,tsh ${ }^{\text {GAL80 }}>$ TNT males resulted in the same copulation duration phenotype, we hypothesized that these 12 neurons (fru-MSNs, Table 2 ) at the genitalia are responsible for the shortened copulation duration phenotype. Since most sensory neurons in Drosophila are cholinergic, we hypothesized that fru-MSNs are the same. Indeed, using Cha-GAL80 that expresses the GAL4 inhibitor in cholinergic neurons eliminated ANN1 expression that originates from the genitalia MSNs (Figure 2D).

\section{Fru MSN neurons co-express doublesex (dsx)}

To rule out the contributions of other neuronal populations to the copulation duration phenotype, we investigated other genetic combinations that target genitalia neurons more specifically. Previous research indicated that the genitalia neurons express $d s x^{11,12,34}$. Indeed, we confirmed that the fru-MSN neurons are also $d s x$ positive (Figure 3A). Replacing fru-GAL4 with $d s x-$ GAL4 in our genetic combination with $\mathrm{FLP}^{335}$ (Dsx ${ }^{\text {GAL4 }}{ }^{\text {,FLP }}{ }^{335}>$ GFP) eliminated all expression in the brain (Figure $3 \mathrm{C}$ ). In the VNC,

Dsx ${ }^{\text {GAL4 }}{ }^{, F L P}{ }^{335}>$ GFP showed consistent expression only in the sexually dimorphic arbors originating 
from the foreleg GRNs and the genitalia neurons (Figure 3B, D, E). Using this highly restrictive genetic combination, we silenced these neurons by TNT expression and evaluated the post-copulatory behaviors. Consistent with our hypothesis, copulation duration was still reduced at the same level for Dsx ${ }^{\text {GAL4 }}$,FLP ${ }^{335}>$ TNT compared to the control (Figure 3G). These results ruled out the involvement of CNS neurons in the copulation duration phenotype and indicate that that the fru neurons responsible for the phenotype are also $d s x$ positive.

The characteristic male-specific midline crossing of the LAN1 arbors that originated from GRNs on the front legs is regulated by both fru and $d s x^{35}$. These GRNs co-express the ion channel ppk25 that is critical for pheromone detection during early courtship steps ${ }^{17}$. To investigate whether the copulation duration phenotype requires female pheromone perception, we paired Dsx ${ }^{\mathrm{GAL}}{ }^{,}, \mathrm{FLP}{ }^{335}>\mathrm{TNT}$ males with females whose pheromone-producing cells were ablated genetically via expression of the pro-apoptotic gene hid under an oenocyte-specific promoter ${ }^{36}$. As expected, the experimental males still exhibited shortened copulation duration compared to the control males, irrespective of whether they were paired with oenocyte-less females (oe-) or their genetic controls (oe+) (Figure 3H). The copulation duration of the control males (TNT-in) is significantly shorter when paired with oe- females compared to control oe+ females (Figure $3 \mathrm{H}$ ). However, no significant effect was observed in the experimental males (TNT); the lack of significance could be due to the variability of the data from the TNT/oe+ pairs. Taken together, these data suggest that the copulation duration phenotype is driven by the silencing of the $f r u / d s x-$ MSNs.

\section{Sensory information detected by fru/dsx neurons is required for copulation persistence}

Next, we asked the biological relevance of a shortened copulation duration as a result of silencing fru/dsx MSNs. In a productive copulation pairing, duration must be long enough for the transfer of sperm and accessory gland fluid. In the three genetic combinations presented here, the median copulation duration for the experimental males is 10-14 minutes, which is longer than the minimal time ( 8 minutes) necessary for sperm transfer ${ }^{25,26}$. To evaluate if sperm is successfully transferred from the test male to the target female, we quantified the number of copulation pairings that resulted in fertilization after a single copulation event between a test male and a virgin Canton-S female. Compared to inactive TNT controls, a significantly lower percentage of males where both copulation duration regulating neural clusters (sAbg- 1 and MSNs) are silenced (Fru ${ }^{\text {GAL4 }}, \mathrm{FLP}^{335}{ }^{\text {,tsh }}{ }^{\mathrm{GAL} 80}>\mathrm{TNT}$ males) could fertilize the virgin females. In contrast, males in which sAbg-1 are not manipulated but MSNs are silenced

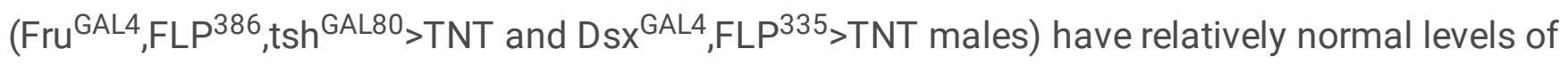
fertilization rates compared to their respective inactive TNT controls (Figure 4A). Therefore, post- 
copulatory fertility is unaffected by the shortened copulation duration due to the silencing of the $\mathrm{fru} / \mathrm{dsx}$ MSNs.

If fertility is unaffected, we wondered if peak copulation persistence is still possible without sensory input from the genitalia. To evaluate if copulation persistence is affected by the silencing of the $f r u / d s x-M S N s$, we applied heat shock as the stress stimulus to copulating flies when copulation persistence is at its peak. We heat-shocked pairs of flies 5 minutes after the onset of copulation and quantified the number of pairs that disengaged. While a large number of pairs in which the fru/ $d s x-M S N s$ in the male genitalia

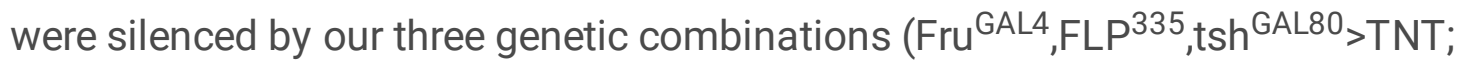
$\mathrm{Fru}^{\mathrm{GAL}}{ }^{,}, \mathrm{FLP}^{386}$, tsh $^{\mathrm{GAL} 80}>\mathrm{TNT}$; Dsx $\left.{ }^{\mathrm{GAL} 4},{ }_{\text {,FLP }}{ }^{335}>\mathrm{TNT}\right)$ terminated copulation, all pairs with the corresponding control males remained copulating (Figure 4B). These results indicate that the $\mathrm{fru} / \mathrm{dsx}$ MSNs are important in maintaining copulation persistence in response to environmental stress before sperm transfer is complete.

\section{Discussion}

Different neuronal populations have been identified to control three distinct aspects of male copulatory behaviors: genital coupling, copulation duration, and sperm transfer ${ }^{26,27,34}$. Here, we have characterized the $f r u / d s x-M S N s$ at the genitalia and identified their novel function in regulating copulation duration and maintaining copulation persistence in the presence of environmental stressors. Although we cannot completely rule out the contribution of pheromone perception in their regulation, the pheromone sensing neurons are not consistently targeted in one of our genetic combination ( $\mathrm{Fru}^{\mathrm{GAL}}{ }^{\mathrm{C}}, \mathrm{FLP}^{386}{ }^{38}, \mathrm{Tsh}^{\mathrm{GAL} 80}>\mathrm{GFP}$ ) (Figure 1B).

Retrograde labeling of various genitalia MSNs have shown that most of these neurons project only to the abdominal ganglion except for one neuron from the surstylus (clasper) that projects all the way to the suboesophageal region of the brain ${ }^{34}$. Since we did not observe any arborizations in the brain, the copulation phenotype appears to be regulated by a subset of the fru/ $d s x^{-} \mathrm{MSNs}$ at the surstylus (claspers) and epandrial ventral lobe (lateral plate) that project specifically to the abdominal ganglion. In addition, the retrograde labeling experiment showed that the axonal terminals of the genitalia neurons juxtapose the dendrites of the abdominal $d s x$ glutamatergic (dsx/vGlut-Abg) and GABAergic (dsx/GABA$\mathrm{Abg}$ ) neurons ${ }^{34}$. Moreover, artificial mechanical stimulation of the genitalia with a minuten pin activated both dsx/vGlut-Abg and dsx/GABA-Abg neurons ${ }^{34}$. Therefore, we can infer that fru/dsx-MSNs neurons make functional synaptic connections to both dsx/vGlut and dsx/GABA neurons in the abdominal ganglion (Figure 5). The shortened copulation duration phenotype when fru/dsx-MSNs are silenced is a result of dsx/vGlut-Abg and/or dsx/GABA-Abg not receiving sensory feedback signals from the genitalia.

dsx/vGlut-Abg are all motor neurons that innervate the phallic and periphallic musculature responsible for genital attachment ${ }^{34}$. On the other hand, dsx/GABA-Abg is comprised of a heterogeneous population of interneurons with different functions. While some of these interneurons inhibit the activity of dsx/vGlut- 
Abg to terminate copulation ${ }^{34}$, others have different functional roles, such as regulating copulation persistence ${ }^{24}$. Therefore, sensory signals received from the fru/dsx-MSNs can influence the relative activity level of dsx/vGlut-Abg and dsx/GABA-Abg neurons with opposing actions and modulate the overall tension of the copulatory muscles. In the absence of sensory information, the baseline muscle strength is sufficient to maintain genital coupling long enough for sperm transfer since males with fru/dsx-MSNs silenced has normal fertility (Figure 4A). However, our results show that the sensory information encoding the male's correct engagement in copulation is necessary to achieve peak copulation persistence before sperm is transferred (Figure 4B, 5). Sensory information - provided by fru/dsx-MSNs - might be a way to measure the quality of the copulation. Genital coupling will lead to bending of the bristle hairs and activate the fru/ $d s x$-MSNs. Which bristle hair gets stimulated and the strength of the stimulation will depend on whether genital coupling is established and the morphology of the female genitalia. For example, an abnormal amount of pressure received by a bristle hair could send less signal to activate the copulatory muscle innervated by dsx-vGlut-Abg. Similarly, a wrong set of bristles bent could activate the dsx-GABA-Abg neurons that inhibit the dsx-vGlut-Abg. A suboptimal activation decreases the total muscular tension during genital coupling. Copulation persistence is a result of the fly's ability to analyze the tradeoff of maintaining copulation during exposure to an environmental stressor. Sensory information that signals the quality of the copulation provides a critical factor in this assessment. The recently published transcriptomic atlas for both the ventral nerve cord ${ }^{37}$ and the male genitalia ${ }^{38}$ provides new possibilities to develop specific neural markers that will allow further investigation of the neural mechanism of how sensory information from genital coupling is encoded and processed in the CNS.

\section{Materials And Methods}

\section{Fly strains}

The following strains were used in this study: fru-GAL4 ${ }^{7}, d s x-G A L 4^{11}, U A S>$ stop $>T N T i n, U A S>$ stop $>T N T^{7}$, $U A S>$ stop $>m C D 8:: G F P^{9}, F L P^{335}$ and $F L P^{386}$ were generated as described in previous study ${ }^{27}$, cha$G A L 80^{39}$, tsh-GAL 80 from Julie Simpson, and Canton-S strain from the Bloomington Stock Center, Bloomington, IN. Oenocyte less (oe-) flies and their controls were generated as previously described ${ }^{36}$.

\section{Immunohistochemistry}

Dissection of 3-7 day old adult flies and immunohistochemistry of the adult nervous system were carried out as described previously ${ }^{40}$ with some modifications in primary and secondary antibodies. Dissection of male reproductive organs was performed on Sylgard plate covered with 1xPBS. To obtain male genitalia, the lower half of the abdomen were dissected and fixed in 4\% PFA at room temperature for 2030 mins. After which the PFA was replaced with 1xPBS. The genitalia were cut out with a pair of microscissors to ensure a flat surface for mounting. Dissected samples were fixed and proceeded with immunostaining as described previously ${ }^{40}$. The following primary and secondary antibodies were used in 
this study: rat polyclonal anti-mCD8 (1:100; Caltag, Burlingame, CA), rabbit anti-GFP (1:600; Invitrogen), mouse anti-GFP (1:500, Life technologies), mouse anti-nc82 (1:20; Hybridoma Bank) ${ }^{41}$, rabbit anti-Dsx ${ }^{\mathrm{M}}$ (1:2000) (kindly supplied by Brian Oliver laboratory, $\mathrm{NIH}$ ), anti-rat IgG conjugated with Alexa Fluor 488 (1:300; Invitrogen), and anti-rabbit IgG conjugated with Alexa Fluor 594 (1:300; Invitrogen).

\section{Microscopy}

Images of the ventral cord, the reproductive tissues, and genitalia were acquired using an Olympus Fluoview FV1000 confocal microscope with a 20X objective. ImageJ was used to stitch the overlapping images together. All other images were acquired using a Zeiss LSM 700 confocal microscope. For the genitalia, lambda stacks from 490-600 nm at $20 \mathrm{~nm}$ intervals were acquired. The autofluorescence signals were unmixed from the $488 \mathrm{~nm}$ signals using the linear unmixing algorithm in the Zen Black software.

\section{Behavioral assays}

Husbandry

Flies were raised on standard cornmeal medium and kept on a $12 \mathrm{hr}: 12 \mathrm{hr}$ day:night cycle at $25^{\circ} \mathrm{C}$ in ambient relative humidity. Each newly eclosed adult was collected and aged for 3-7 days in an isolation vial $(16 \times 100-\mathrm{mm}$ ) supplied with $\sim 2 \mathrm{ml}$ of fly food. Virgin, wildtype Canton-S females were aged in groups of 20-40 for 3-7 days. All behavioral experiments were performed at $25^{\circ} \mathrm{C}$ with $\sim 50 \%$ humidity during the first $3 \mathrm{hrs}$. after lights on.

\section{Copulation Assay}

Copulation assays were performed in 12-well plates (Thermo Scientific BioLite Multidish). A square glass plate covering four wells was used as the lid. Assays were performed in 4 wells with $\sim 5 \mathrm{ml}$ standard fly food to maintain humidity in each well. An experimental male was paired with a virgin female and the courtship behavior was videotaped for at least an hour. If a pair did not copulate within an hour, it was considered unsuccessful. Copulation duration was calculated from the beginning of genital coupling until the male was dismounted from the female.

\section{Fertility Assay}

Freshly hatched males were isolated into a glass tube $(16 \times 100 \mathrm{~mm})$ with $2 \mathrm{ml}$ of food and kept for 4-5 days at standard conditions $\left(25^{\circ} \mathrm{C}, 50 \% \mathrm{RH}\right)$. A 6-7 day old CS virgin female was introduced into each tube using an aspirator. After transferring the vials back to standard conditions, the flies were allowed to interact for $1 \mathrm{~h}$ and observed every 10 mins for successful copulation. Tubes with pairs that did not copulate were discarded. For the rest of the tubes, males were removed by quick anaesthetization and females were allowed to lay eggs for $24 \mathrm{~h}$ and then discarded. Fertility was recorded after 7 days by observing the presence of progeny. 
A single experimental male was paired with a wildtype CS virgin female in a glass tube $(16 \times 100 \mathrm{~mm})$ with or without food. If copulated in a vial with food, the pairs were transferred to an empty vial before heat shock to ensure efficient heat transfer. Each pair that successfully copulated (within 5 mins) was subjected to heat stress by submersing the empty glass vial $(16 \times 100 \mathrm{~mm})$ in a $37^{\circ} \mathrm{C}$ water bath for 30 seconds. Frequency of copulation termination was recorded.

\section{References}

1. Burtis, K. C. \& Baker, B. S. Drosophila doublesex gene controls somatic sexual differentiation by producing alternatively spliced mRNAs encoding related sex-specific polypeptides., 56, 997-1010 https://doi.org/doi:10.1016/0092-8674(89)90633-8 (1989).

2. Ito, H. et al. Sexual orientation in Drosophila is altered by the satori mutation in the sex-determination gene fruitless that encodes a zinc finger protein with a BTB domain. Proc Natl Acad Sci U SA, 93, 9687-9692 (1996).

3. Ryner, L. C. et al. Control of male sexual behavior and sexual orientation in Drosophila by the fruitless gene., 87, 1079-1089 (1996). doi:S0092-8674(00)81802-4 [pii]

4. Lee, G. et al. Spatial, temporal, and sexually dimorphic expression patterns of the fruitless gene in the Drosophila central nervous system. J Neurobiol, 43, 404-426 https://doi.org/doi:10.1002/10974695(20000615)43:4<404::AID-NEU8>3 (2000). 0.C0;2-D [pii]

5. Demir, E. \& Dickson, B. J. fruitless splicing specifies male courtship behavior in Drosophila. Cell 121, 785-794, doi:S0092-8674(05)00407-1 [pii]10.1016/j.cell.2005.04.027 (2005)

6. Manoli, D. S. et al. Male-specific fruitless specifies the neural substrates of Drosophila courtship behaviour., 436, 395-400, doi:nature03859 (2005). [pii]10.1038/nature03859

7. Stockinger, P., Kvitsiani, D., Rotkopf, S., Tirian, L. \& Dickson, B. J. Neural circuitry that governs Drosophila male courtship behavior. Cell 121, 795-807, doi:S0092-8674(05)00406-X [pii]10.1016/j.cell. 2005.04.026 (2005)

8. Cachero, S., Ostrovsky, A. D., Yu, J. Y., Dickson, B. J. \& Jefferis, G. S. Sexual Dimorphism in the Fly Brain. Curr Bio/ 20, 1589-1601, doi:S0960-9822(10)00947-4 [pii]10.1016/j.cub. 2010.07 .045 (2010)

9. Yu, J. Y., Kanai, M. I., Demir, E., Jefferis, G. S. \& Dickson, B. J. Cellular Organization of the Neural Circuit that Drives Drosophila Courtship Behavior. Curr Biol 20, 1602-1614, doi:S09609822(10)01015-8 [pii]10.1016/j.cub. 2010.08.025 (2010)

10. Rideout, E. J., Billeter, J. C. \& Goodwin, S. F. The sex-determination genes fruitless and doublesex specify a neural substrate required for courtship song. Curr Biol, 17, 1473-1478 https://doi.org/doi:10.1016/j.cub.2007.07.047 (2007).

11. Rideout, E. J., Dornan, A. J., Neville, M. C., Eadie, S. \& Goodwin, S. F. Control of sexual differentiation and behavior by the doublesex gene in Drosophila melanogaster. Nat Neurosci 13, 458-466, doi:nn.2515 [pii]10.1038/nn.2515 (2010) 
12. Robinett, C. C., Vaughan, A. G., Knapp, J. M. \& Baker, B. S. Sex and the single cell. II. There is a time and place for sex. PLoS Biol, 8, e1000365 https://doi.org/doi:10.1371/journal.pbio.1000365 (2010).

13. Villella, A. \& Hall, J. C. Courtship anomalies caused by doublesex mutations in Drosophila melanogaster., 143, 331-344 (1996).

14. Pan, Y. \& Baker, B. S. Genetic identification and separation of innate and experience-dependent courtship behaviors in Drosophila., 156, 236-248 https://doi.org/doi:10.1016/j.cell.2013.11.041 (2014).

15. Yamamoto, D. \& Koganezawa, M. Genes and circuits of courtship behaviour in Drosophila males. Nat Rev Neurosci, 14, 681-692 https://doi.org/doi:10.1038/nrn3567 (2013).

16. Kurtovic, A., Widmer, A. \& Dickson, B. J. A single class of olfactory neurons mediates behavioural responses to a Drosophila sex pheromone. Nature 446, 542-546, doi:nature05672 [pii]10.1038/nature05672 (2007)

17. Thistle, R., Cameron, P., Ghorayshi, A., Dennison, L. \& Scott, K. Contact Chemoreceptors Mediate MaleMale Repulsion and Male-Female Attraction during Drosophila Courtship. Cell 149, 1140-1151, doi:S0092-8674(12)00523-5 [pii]10.1016/j.cell.2012.03.045 (2012)

18. Shankar, S. et al. The neuropeptide tachykinin is essential for pheromone detection in a gustatory neural circuit. eLife, 4, e06914 https://doi.org/doi:10.7554/elife.06914 (2015).

19. Ejima, A. \& Griffith, L. C. Courtship initiation is stimulated by acoustic signals in Drosophila melanogaster. PLoS One, 3, e3246 https://doi.org/doi:10.1371/journal.pone.0003246 (2008).

20. Kohatsu, S. \& Yamamoto, D. Visually induced initiation of Drosophila innate courtship-like following pursuit is mediated by central excitatory state. Nat Commun, 6, 6457 https://doi.org/doi:10.1038/ncomms7457 (2015).

21. Rice, G. et al. A standardized nomenclature and atlas of the male terminalia of Drosophila melanogaster., 13, 51-64 https://doi.org/doi:10.1080/19336934.2019.1653733 (2019).

22. Jagadeeshan, S. \& Singh, R. S. A time-sequence functional analysis of mating behaviour and genital coupling in Drosophila: role of cryptic female choice and male sex-drive in the evolution of male genitalia. J Evol Biol 19, 1058-1070, doi:JEB1099 [pii]10.1111/j.1420-9101. 2006.01099.x (2006)

23. Taylor, B. J. Sexually dimorphic neurons of the terminalia of Drosophila melanogaster: II. Sex-specific axonal arborizations in the central nervous system. J Neurogenet, 5, 193-213 (1989).

24. Crickmore, M. A. \& Vosshall, L. B. Opposing dopaminergic and GABAergic neurons control the duration and persistence of copulation in Drosophila., 155, 881-893 https://doi.org/doi:10.1016/j.cell.2013.09.055 (2013).

25. Latham, K. L., Liu, Y. S. \& Taylor, B. J. A small cohort of FRU(M) and Engrailed-expressing neurons mediate successful copulation in Drosophila melanogaster. BMC Neurosci, 14, 57 https://doi.org/doi:10.1186/1471-2202-14-57 (2013).

26. Tayler, T. D., Pacheco, D. A., Hergarden, A. C., Murthy, M. \& Anderson, D. J. A neuropeptide circuit that coordinates sperm transfer and copulation duration in Drosophila. Proc Natl Acad Sci U S A, 109, 20697-20702 https://doi.org/doi:10.1073/pnas.1218246109 (2012). 
27. Jois, S., Chan, Y. B., Fernandez, M. P. \& Leung, A. K. Characterization of the Sexually Dimorphic fruitless Neurons That Regulate Copulation Duration. Front Physiol, 9, 780 https://doi.org/doi:10.3389/fphys.2018.00780 (2018).

28. Beaver, L. M. \& Giebultowicz, J. M. Regulation of copulation duration by period and timeless in Drosophila melanogaster. Curr Biol, 14, 1492-1497 https://doi.org/doi:10.1016/j.cub.2004.08.022S0960982204006050 (2004). [pii]

29. Lewis, Z., Heys, C., Prescott, M. \& Lize, A. You are what you eat., 5, 541-543 https://doi.org/doi:10.4161/gmic.29153 (2014).

30. Brand, A. H. \& Perrimon, N. Targeted gene expression as a means of altering cell fates and generating dominant phenotypes. Development, 118, 401-415 (1993).

31. Alekseyenko, O. V. et al. Single serotonergic neurons that modulate aggression in Drosophila. Curr Biol, 24, 2700-2707 https://doi.org/doi:10.1016/j.cub.2014.09.051 (2014).

32. Alekseyenko, O. V., Chan, Y. B., Li, R. \& Kravitz, E. A. Single dopaminergic neurons that modulate aggression in Drosophila. Proc Natl Acad Sci U S A 110, 6151-6156, doi:1303446110 [pii]10.1073/pnas.1303446110 (2013)

33. Kerwin, P., Yuan, J. \& von Philipsborn, A. C. Female copulation song is modulated by seminal fluid. Nat Commun, 11, 1430 https://doi.org/doi:10.1038/s41467-020-15260-6 (2020).

34. Pavlou, H. J. et al. Neural circuitry coordinating male copulation. eLife, 5 , https://doi.org/doi:10.7554/eLife.20713 (2016).

35. Mellert, D. J., Knapp, J. M., Manoli, D. S., Meissner, G. W. \& Baker, B. S. Midline crossing by gustatory receptor neuron axons is regulated by fruitless, doublesex and the Roundabout receptors. Development 137, 323-332, doi:137/2/323 [pii]10.1242/dev.045047 (2010)

36. Billeter, J. C., Atallah, J., Krupp, J. J., Millar, J. G. \& Levine, J. D. Specialized cells tag sexual and species identity in Drosophila melanogaster., 461, 987-991 https://doi.org/doi:10.1038/nature08495 (2009).

37. Allen, A. M. et al. A single-cell transcriptomic atlas of the adult Drosophila ventral nerve cord. eLife, 9, https://doi.org/doi:10.7554/eLife.54074 (2020).

38. Vincent, B. J. et al. An Atlas of Transcription Factors Expressed in Male Pupal Terminalia of Drosophila melanogaster. G3 9, 3961-3972, doi:10.1534/g3.119.400788 (2019)

39. Kitamoto, T. Conditional disruption of synaptic transmission induces male-male courtship behavior in Drosophila. Proc Natl Acad Sci U S A, 99, 13232-13237

https://doi.org/doi:10.1073/pnas.202489099 (2002).

40. Mundiyanapurath, S., Chan, Y. B., Leung, A. K. \& Kravitz, E. A. Feminizing cholinergic neurons in a male Drosophila nervous system enhances aggression. Fly (Austin), 3, 179-184, doi:8989 (2009). [pii]

41. Hofbauer, A. et al. The Wuerzburg hybridoma library against Drosophila brain. J Neurogenet, 23, 7891, doi:907468467 (2009). [pii]10.1080/01677060802471627 


\section{Declarations}

Acknowledgments

We thank our mentor Edward Kravitz for providing the resources to initiate this project. We thank Barry Dickson, Liqun Luo, Julie Simpson, Joel Levine, Joshua Krupp and the Bloomington Stock Centre for fly stocks; Sarah Parker for statistics advice, and past members of the Kravitz laboratory for helpful discussion (Olga Alekseyenko, Sarah Certel, Jill Penn, and Joanne Yew).

Author Contributions

$A L, Y C$, and MF conceived and designed the experiments. AL, YC, SJ, NP carried out the experiments. AL led the project. AL wrote the manuscript. LJ helped with revision. All authors provided critical feedback and helped shape the research, analysis and manuscript.

Financial Disclosure Statement

This study was supported by the Natural Sciences and Engineering Research Council of Canada (NSERC), [funding reference number RGPIN-2014-06012]. Cette recherche a été finance par le Conseil de recherches en sciences naturelles et en genie du Canada (CRSNG), [numéro de reference RGPIN-2014-06012], Canadian Foundation for Innovation, John R. Evans Leaders Opportunity Fund, and internal funding support from the Western College of Medicine and College of Medicine, University of Saskatchewan [Adelaine K. Leung (Principal Investigator)]. These funders played no role in the study design, data collection and analysis, decision to publish, or preparation of the manuscript.

\section{Tables}

Table 1. Summary of fru expression restricted by FLP ${ }^{335}$ or FLP $\mathrm{P}^{386}$ in combination with fruGAL4 and tsh-GAL80. 


\begin{tabular}{|l|l|c|c|}
\hline Neurons & Arbors & FLP335 & FLP386 \\
\hline ORNs (Peripheral) & DA1 (Brain) & + & + \\
\hline & VA1v (Brain) & + & + \\
\hline & VL2a (Brain) & + & + \\
\hline JONs (Peripheral) & AMMC (Brain) & + & + \\
\hline MSNs (Peripheral) & ANN1 (VNC) & + & + \\
\hline GRNs (Peripheral) & LAN1 (VNC) & + & + \\
\hline$\sim 5$ 5HT/DA Abg-1 (VNC) & $\begin{array}{l}\text { Reproductive tissues } \\
\text { (Peripheral) }\end{array}$ & + & \\
\hline $\begin{array}{l}\sim 12 \text { AbgNs uncharacterized } \\
(\text { VNC) }\end{array}$ & & & + \\
\hline$\sim 8$ AbgNs uncharacterized (VNC) & & & + \\
\hline
\end{tabular}

In brackets is the anatomical location of the neurons or arbors. ORN, olfactory receptor neuron; JON, Johnston's organ neuron; MSN, mechanosensory neuron; GRN, gustatory receptor neuron; AbgN, abdominal neuron; 5HT, serotonergic neuron; DA, dopaminergic neuron; VNC, ventral nerve cord.

Table 2. Expression Summary of the Male Genitalia of the Different Genetic Combinations.

\begin{tabular}{|c|c|c|c|c|c|c|c|c|c|}
\hline \multicolumn{2}{|c|}{ Transgene Present } & \multicolumn{2}{|c|}{$\begin{array}{c}\text { Epandrial Ventral Lobe } \\
\text { (EVL) }\end{array}$} & \multicolumn{2}{c|}{ Surstylus (SUR) } & \multicolumn{2}{c|}{ Cercus (CER) } & n \\
\hline FLP & fru-Gal4 & $\begin{array}{c}\text { Tsh- } \\
\text { Gal80 }\end{array}$ & $\begin{array}{c}\text { Percent } \\
\text { Sample } \\
\text { Showing } \\
\text { Expression } \\
\text { (\%) }\end{array}$ & $\begin{array}{c}\text { Average } \\
\text { Number } \\
\text { of Cells }\end{array}$ & $\begin{array}{c}\text { Percent } \\
\text { Sample } \\
\text { Showing } \\
\text { Expression } \\
(\%)\end{array}$ & $\begin{array}{c}\text { Average } \\
\text { Number } \\
\text { of Cells }\end{array}$ & $\begin{array}{c}\text { Percent } \\
\text { Sample } \\
\text { Showing } \\
\text { Expression } \\
(\%)\end{array}$ & $\begin{array}{c}\text { Average } \\
\text { Number of } \\
\text { Cells }\end{array}$ \\
\hline- & - & - & - & $20^{*} \pm 0.34$ & & $25^{*} \pm 0.63$ & & $32.7^{*} \pm 1.56$ & - \\
\hline- & + & - & 100 & $10 \pm 4$ & 100 & $16 \pm 2$ & 100 & $20 \pm 2$ & 12 \\
\hline 335 & + & + & 0 & - & 100 & $10 \pm 3$ & 100 & $7 \pm 2$ & 10 \\
\hline 386 & + & + & 0 & - & 94 & $8 \pm 3$ & 88 & $4 \pm 2$ & 16 \\
\hline
\end{tabular}

*Based on bristle count published in Taylor 1998.

\section{Figures}


A

$\mathrm{Fru}^{\mathrm{GAL}}{ }^{\mathrm{G}}, \mathrm{FLP}^{335}, \mathrm{tsh}^{\mathrm{GAL} 80}>\mathrm{GFP}$
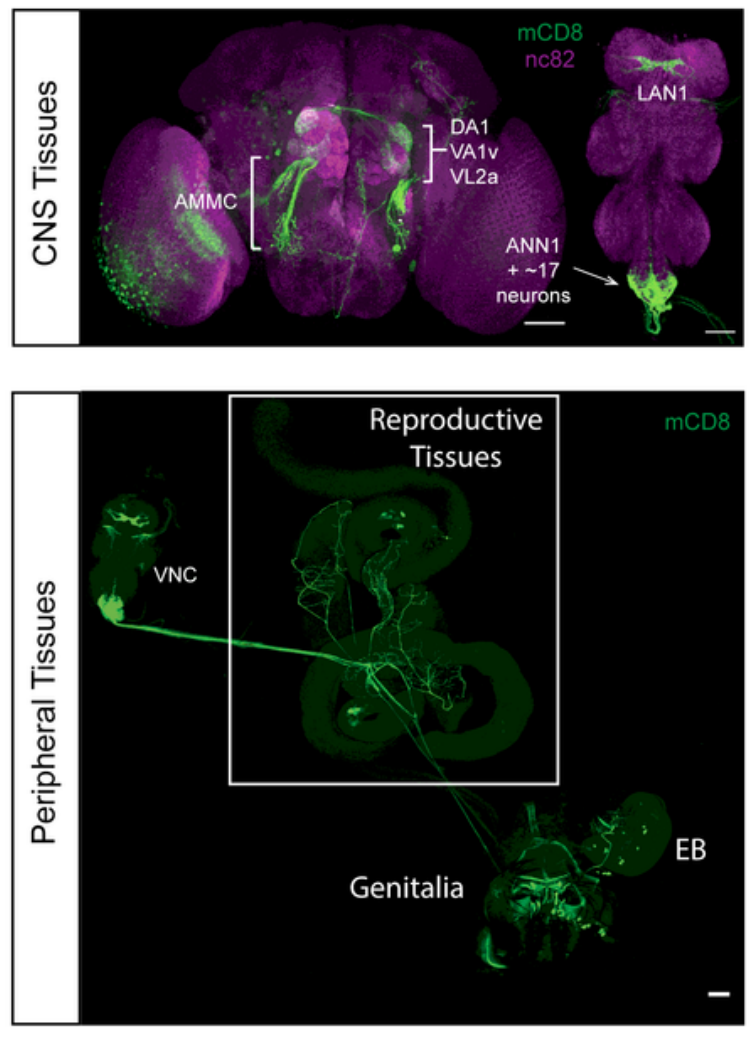

C

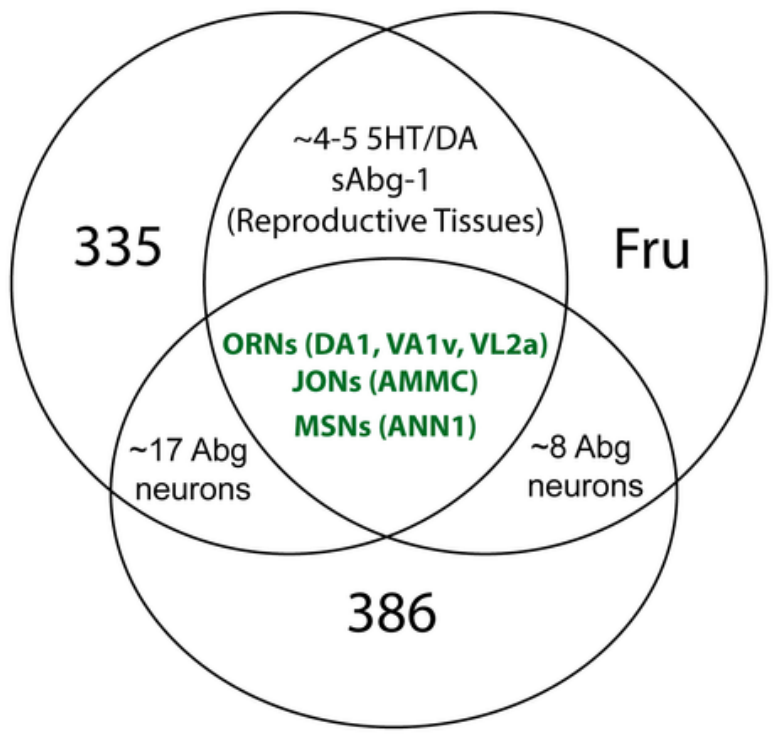

B

$\mathrm{Fru}^{\mathrm{GAL} 4}, \mathrm{FLP}^{386}, \mathrm{tsh}^{\mathrm{GAL80}}>\mathrm{GFP}$
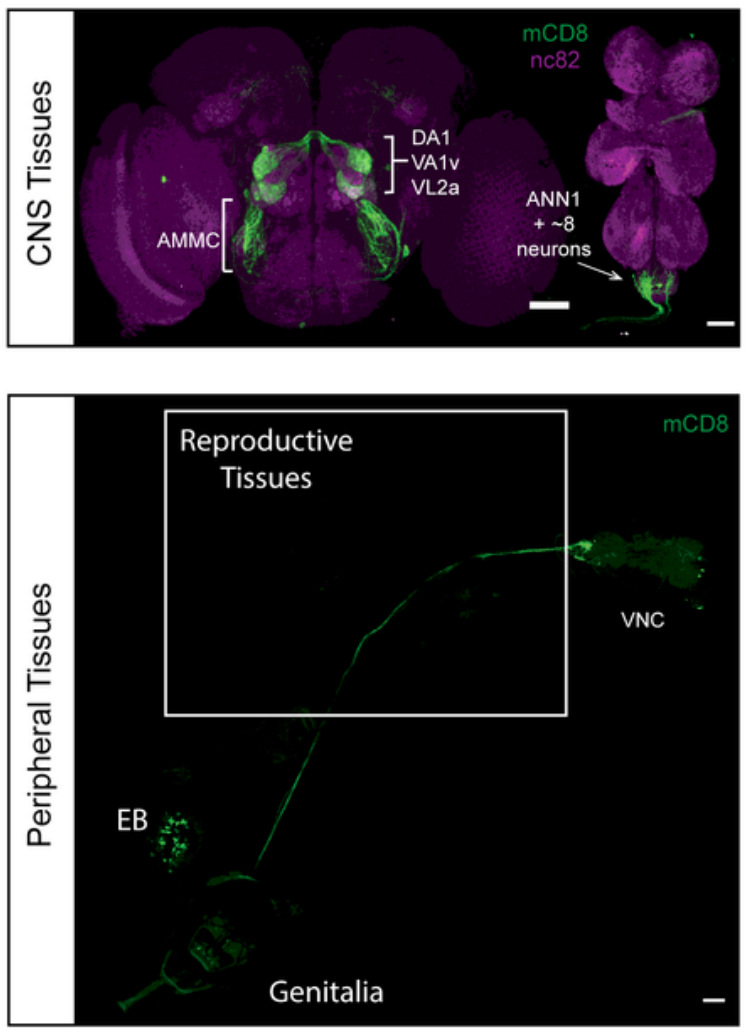

D

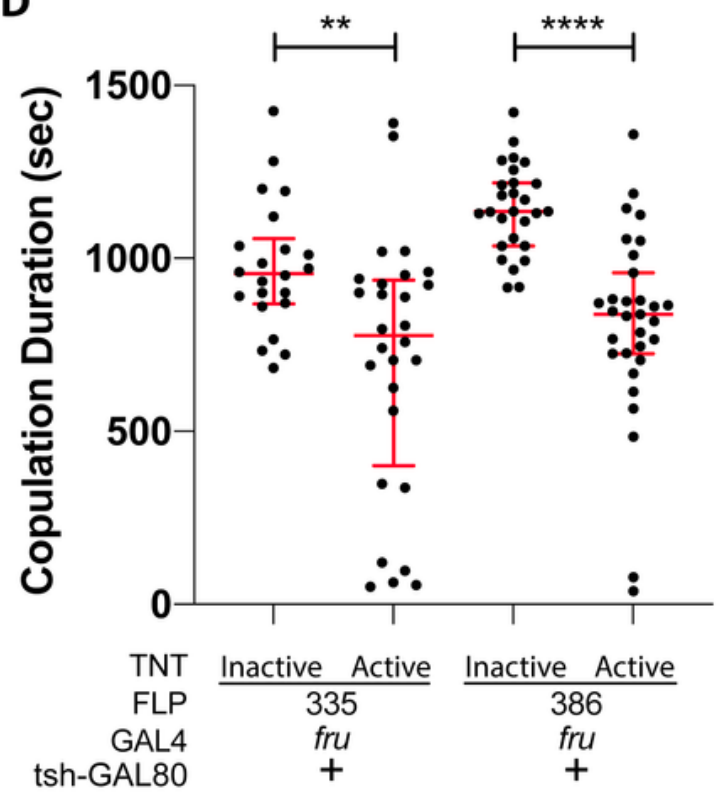

\section{Figure 1}

Different groups of fru neurons regulate copulation duration. A, B) Comparison of fru neuronal expression restricted by two different FLP lines. Expression profile of FruGAL4,FLP335,tshGAL80>GFP (A) is compared to that of FruGAL4,FLP386,tshGAL80>GFP (B). CNS tissues were stained with anti-mCD8 (green) and nc82 (magenta) and peripheral tissues were stained with anti-mCD8 (green) only. Expression in the reproductive tissues was notably eliminated via FLP386. Scale Bar $=50 \mu \mathrm{m}$. C) Venn diagrams 
highlighting the common fru neurons targeted by both FLP lines as shown in A) and B). Names of the arbors are in brackets followed by the names of the neuronal cluster where they are originated. Three common fru neurons were consistently labeled by both FLP lines: 1) the sexually dimorphic projections in the glomeruli from fru ORNs (DA1, VA1v, VL2a), 2) projections in the antennal mechanosensory motor complex (AMMC) from fru JONs, 3) projections in the abdominal ganglion (ANN1) from fru MSNs in the genitalia. See also Table 1 in text. (D) Effect of silencing FLP335 and FLP386 restricted labeling of fruGAL4. A shortened copulation duration was observed in both genetic combinations, therefore, the common neuronal clusters targeted by both FLP lines (ORNs, JONs, MSNs) were responsible for the copulation phenotype. Black dots are individual values. Red lines indicate the median with interquartile range $(n=22-31) .{ }^{* \star} p<0.0034,{ }^{\star \star * \star} p<0.0001$ by Mann Whitney test. 
A

$D$

$\vee$

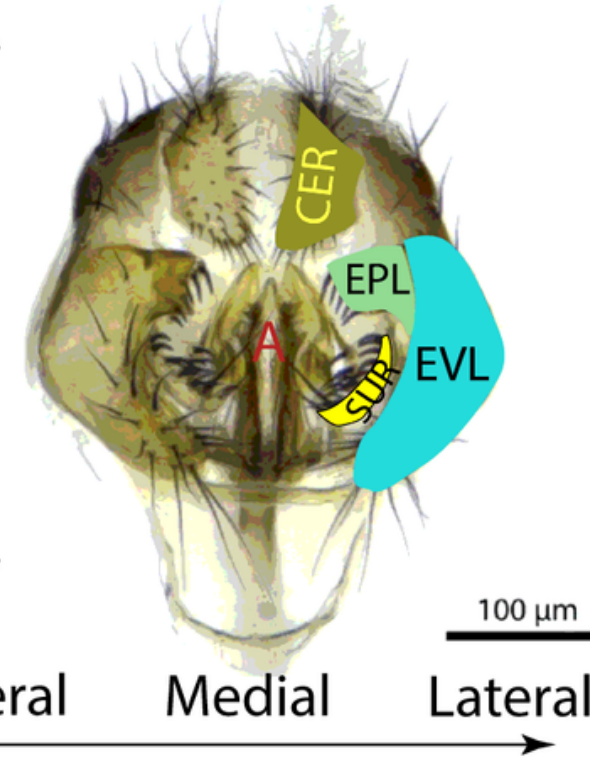

C

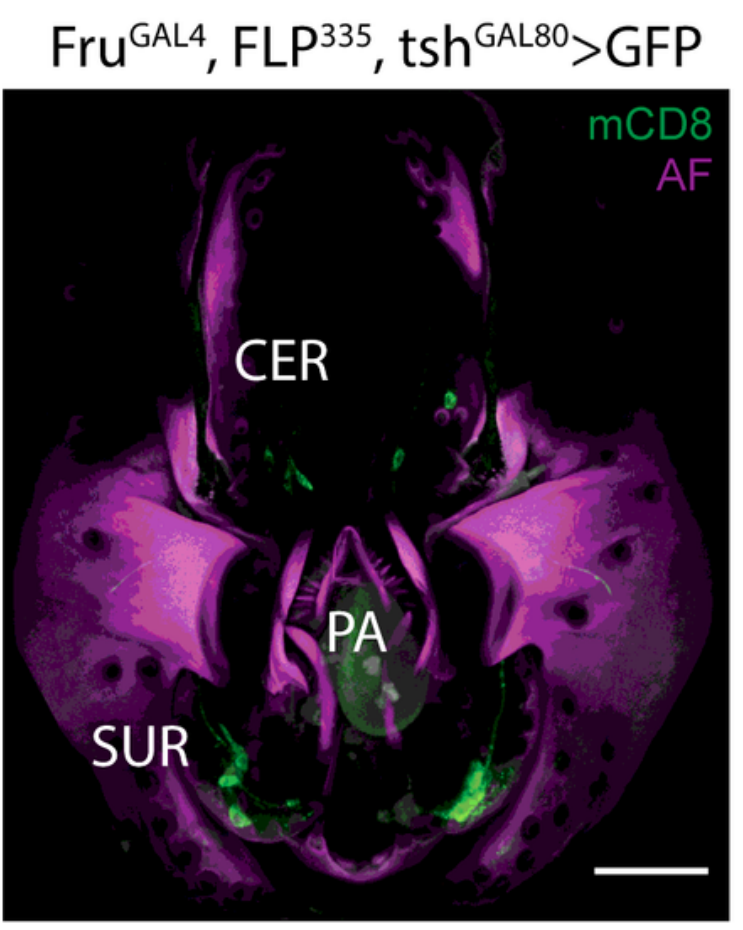

B

fru-GAL4>GFP

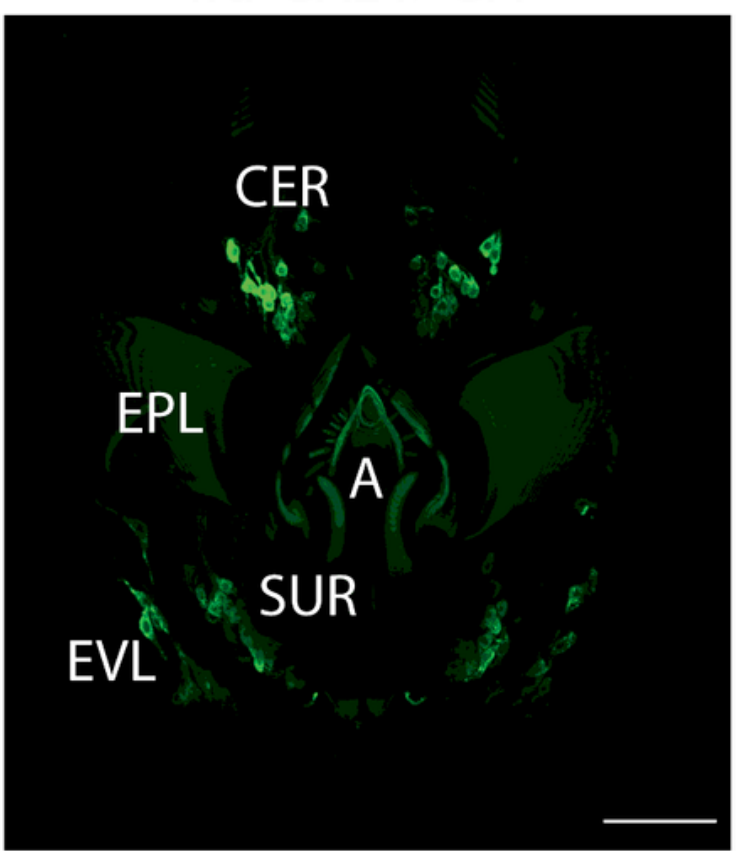

D

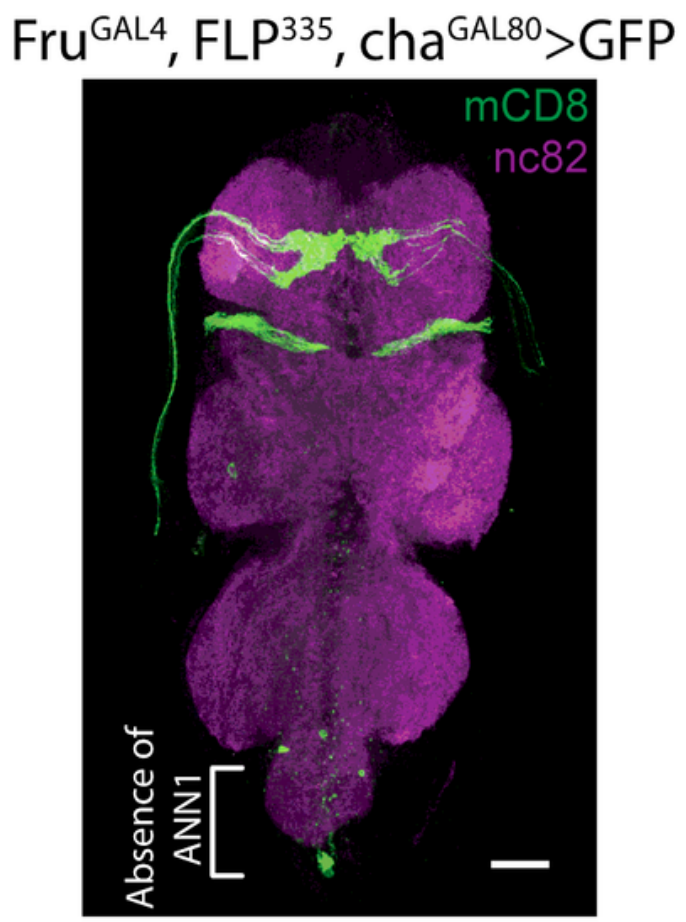

Figure 2

Characterization of fru neurons in the male genitalia. A) Light microscope image of a male genitalia adapted from a previous work 21 . The male genitalia are comprised of the periphallic structures: cercus (CER), surstylus (SUR), epandrial ventral lobe (EVL) and the phallic structures: aedeagus (A). The phallic structures are attached to copulatory muscles (Figure 5) and located just anterior to the periphallic structures. Stereotypical bristles on the surface of these cuticular plates are innervated by 
mechanosensory neurons (MSNs)23. B) fru MSNs at the male genitalia. Expression profile of fru-GAL4 driving UASmCD8::GFP in the male genitalia. fru expression was observed in $~ 50 \%$ of MSNs at the epandrial ventral lobe, $\sim 64 \%$ of MSNs at the surstylus, and $\sim 61 \%$ of MSNs at the cercus (Table 2). C) fru MSNs restricted by FLP335. Expression profile of fru-GAL4, UAS>stop>mCD8::GFP, FLP335, tsh-GAL80 in the male genitalia. Adding FLP335 eliminated GFP expression in the epandrial ventral lobe and restricted expression at the surstylus and the cercus (Table 2). The autofluorescence and 488 signals were unmixed as described in Methods. D) fru MSNs are cholinergic. Expression profile of

FruGAL4,FLP335,ChaGAL80>GFP in the male ventral nerve cord. Adding the transgene cha-Gal80, which inhibits GAL4 in cholinergic neurons, eliminated ANN1 expression that originated from the genitalia MSNs (compare to Figure 1A, B). Only fru-Abg-1 and other neurons in the abdominal ganglion showed expression. Scale Bar $=50 \mu \mathrm{m}$. 
A

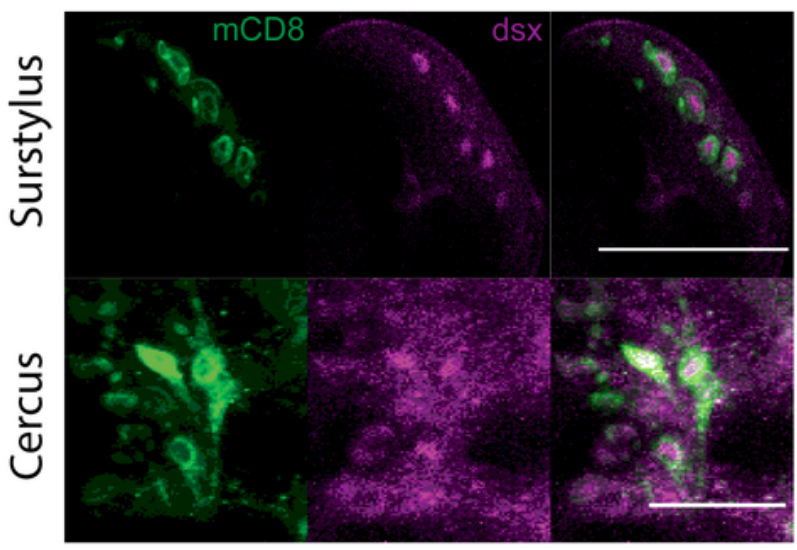

C

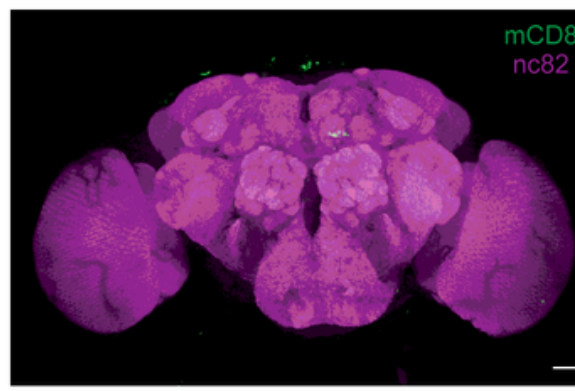

G

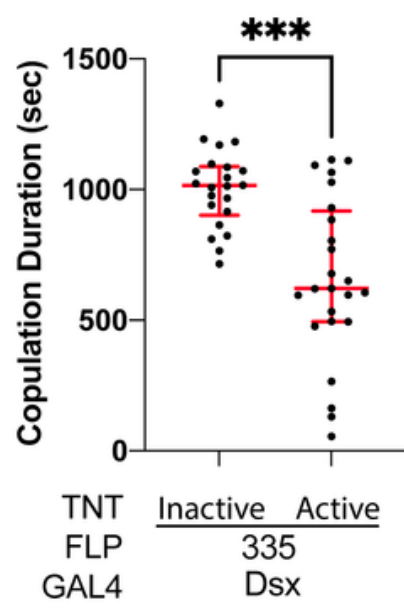

H
B

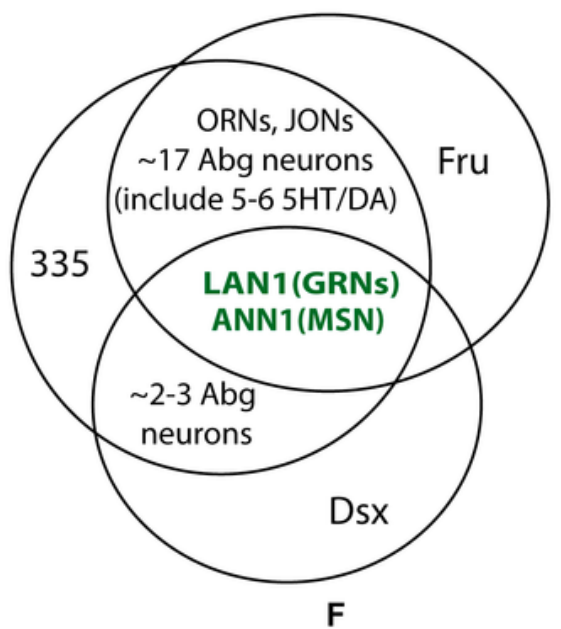

E

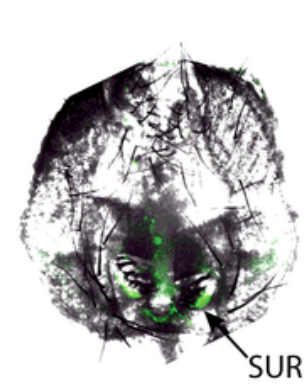

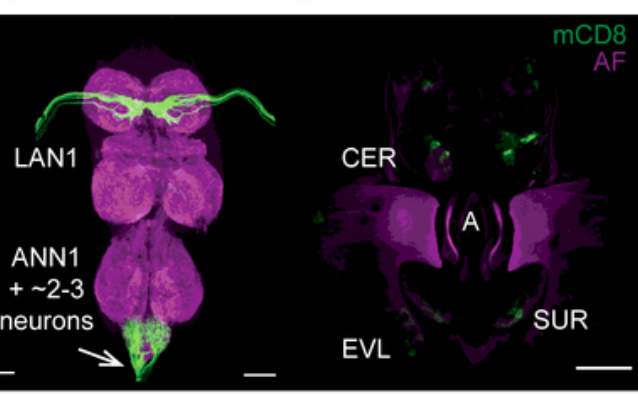

ns

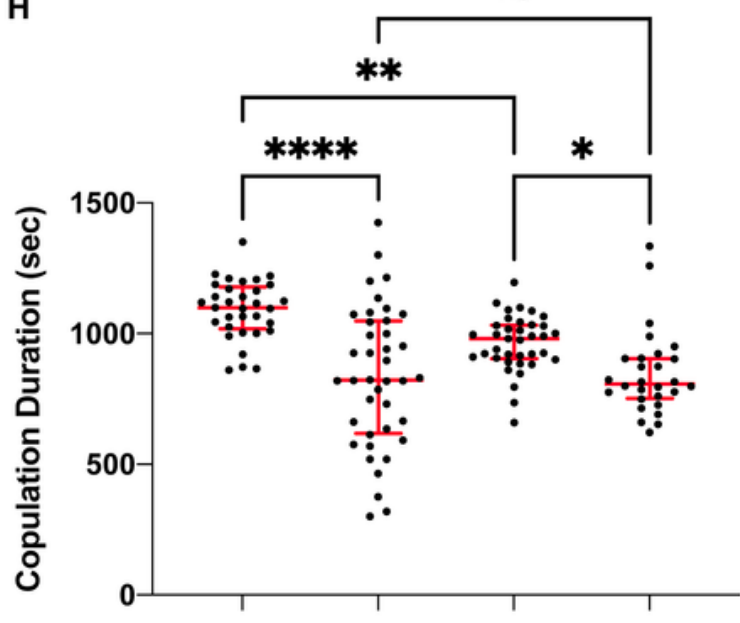

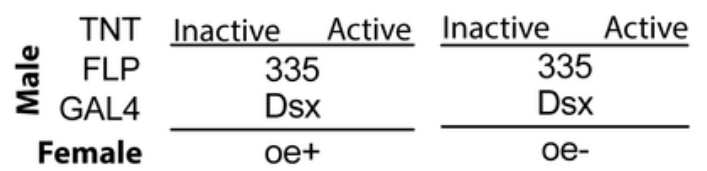

\section{Figure 3}

fru/dsx MSNs regulates copulation duration. A) fru MSNs co-express dsx. Expression profile of FruGAL4,FLP335>FLP in the male genitalia. Double staining with anti-mCD8 (green) and anti-dsx (magenta) showed coexpression of dsx in fru-MSNs at the surstylus and the cercus of the genitalia. Scale Bar $=50 \mu \mathrm{m}$. B) Venn diagram summarizing fru and dsx expression pattern restricted by FLP335. The sexually dimorphic LAN1 and ANN1 arbors originated from the GRNs on the front legs and MSNs of the 
genitalia were the only common expression observed in both GAL4 lines. C-F) Expression profile of DsxGAL4,FLP335>GFP. The CNS tissues were stained with anti-mCD8 (green) and anti-nc82 (magenta). No expression was observed in the brain (C), and the ventral nerve cord showed expression for the sexually dimorphic LAN1 arbors that originated from the GRNs in the front legs and the sexually dimorphic ANN1 arbors that originated from the MSNs in the genitalia (D). E) The genitalia were stained with anti-mCD8 (green), autofluorescence (magenta). Expression was observed in the MSNs located on CER, SUR, and EVL of the genitalia. Scale Bar $=50 \mu \mathrm{m}$. F) GFP expression on the genitalia overlaid with a DIC image of the cuticle. F) Effect of silencing FLP335 restricted labeling of dsx-GAL4. The copulation duration was shortened by $\sim 1.6$ fold in the experimental line compared to the control (DsxGAL4,FLP335>TNTin: n=22; DsxGAL4,FLP335 >TNT: $n=24$; black dots are individual values; red lines indicate the median with interquartile range; ${ }^{*} p<0.0029,{ }^{\star \star \star} p<0.0001$ by Mann Whitney test). $G$ ) Pheromone requirement in the regulation of copulation duration. Mating pairs were set up between males expressing TNT in FLP335 restricted dsx neurons (TNT and TNT-in=inactive control) and either females with ablated oenocytes (oe-) or their genetic controls (oe+). When paired with control females, the copulation duration was shortened by 1.9-fold for the experimental line (active TNT) compared to the control line with an inactive TNT expressed in the subset of dsx neurons restricted by FLP335 (FLP335, dsx>TNTin: $n=33$; FLP335, dsx>TNT: $n=40$ ). Black dots indicate individual values; red lines indicate the median with interquartile range. One-way ANOVA Kruskal-Wallis, adjusted $p$ value at $<0.0001$ using Dunn's multiple comparisons test). When paired with oe- females, copulation duration was shortened by 1.2-fold in the experimental males compared to the control males (DsxGAL4,FLP335>TNTin: $n=37$; DsxGAL4,FLP335>TNT: $n=28$; One-way ANOVA Kruskal-Wallis, adjusted $p$ value at $<0.0289$ using Dunn's multiple comparisons test). When paired with control males (DsxGAL4,FLP335>TNTin), oe- females led to a small but significant reduction of 1.1-fold in copulation duration compared to the oe+ control (DsxGAL4,FLP335>TNTin vs oe+: $n=33$, DsxGAL4,FLP335>TNTin vs. oe-: $n=37$; One-way ANOVA KruskalWallis, adjusted $p$ value at $<0.0088$ using Dunn's multiple comparisons test). When paired with experimental males (DsxGAL4,FLP335>TNT), no significant difference was observed between oe+ and oe- females (DsxGAL4,FLP335>TNT vs oe+: $n=40$, DsxGAL4,FLP335>TNT vs. oe-: $n=28$; One-way ANOVA Kruskal-Wallis, adjusted $p$ value at $>0.9999$, Dunn's multiple comparisons test).

A

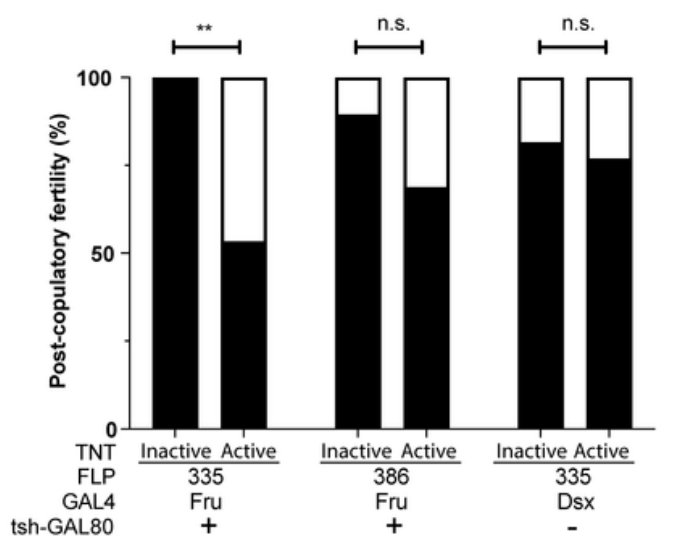

B

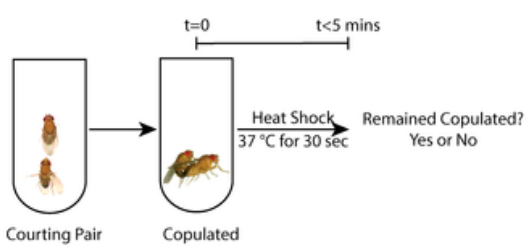

C

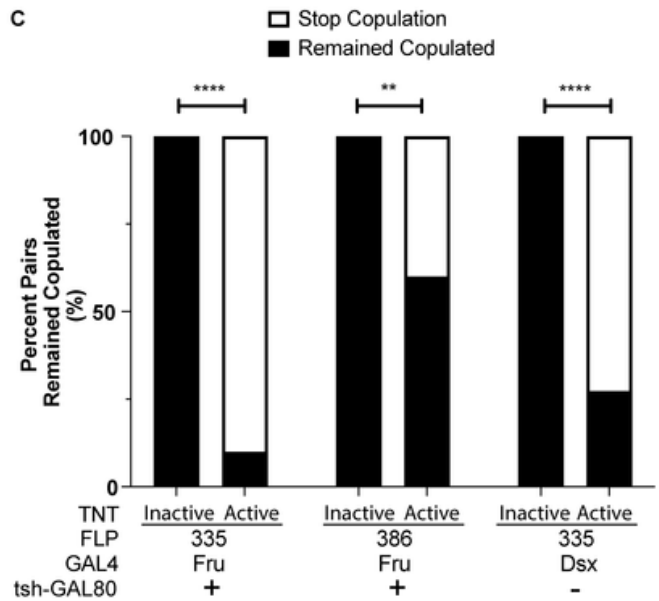

Figure 4 
Sensory information detected by fru/dsx-MSNs is necessary to maintain copulation persistence. Mating pairs were set up between males expressing TNT in either FLP335 or FLP386 restricted dsx or fru neurons and CS females. A) The fertility of the female after a single copulation event was assessed. Percentage of females that produced progeny (FruGAL4,FLP335,tshGAL80>TNTin: $n=15$;

FruGAL4,FLP335,tshGAL80>TNT: n=15; FruGAL4,FLP386,tshGAL80>TNTin: n=19;

FruGAL4,FLP386,tshGAL80>TNT: $n=16$; DsxGAL4,FLP335>TNTin: $n=27$; DsxGAL4,FLP335>TNT: $n=26$ ) n.s. $=$ not significant, ${ }^{\star \star} \mathrm{p}=0.0063$ by Fisher's exact test. Females mated with males of FruGAL4,FLP335,tshGAL80>TNT, which included both copulation duration regulating neural clusters (frusAbg-1 and fru/dsx-MSNs) were less likely to produce progeny compared to the TNT-in controls. On the other hand, females mated with males of FruGAL4,FLP386,tshGAL80>TNT or dsxGAL4,FLP335>TNT, both of which include only one copulation duration regulating neural cluster (fru/dsx-MSNs) had normal fertility. B) Copulation persistence was assessed by heat shocking copulating pairs within the first 5 mins of copulation initiation. Percentage of mating pairs that remained copulated during heat shock (FruGAL4,FLP335,tshGAL80>TNTin: n=13; FruGAL4,FLP335,tshGAL80>TNT: n=10; FruGAL4,FLP386,tshGAL80>TNTin: $n=18$; FruGAL4,FLP386,tshGAL80>TNT: $n=15$; DsxGAL4,FLP335>TNTin: $n=30$; DsxGAL4,FLP335>TNT: $n=22) * \star * * p<0.0001, * * p=0.0045$, by Fisher's exact test. TNT-silencing of neurons by the three genetic combinations all resulted in higher percentage of males that terminated copulation upon heat shock compared to their respective TNT-in controls. 


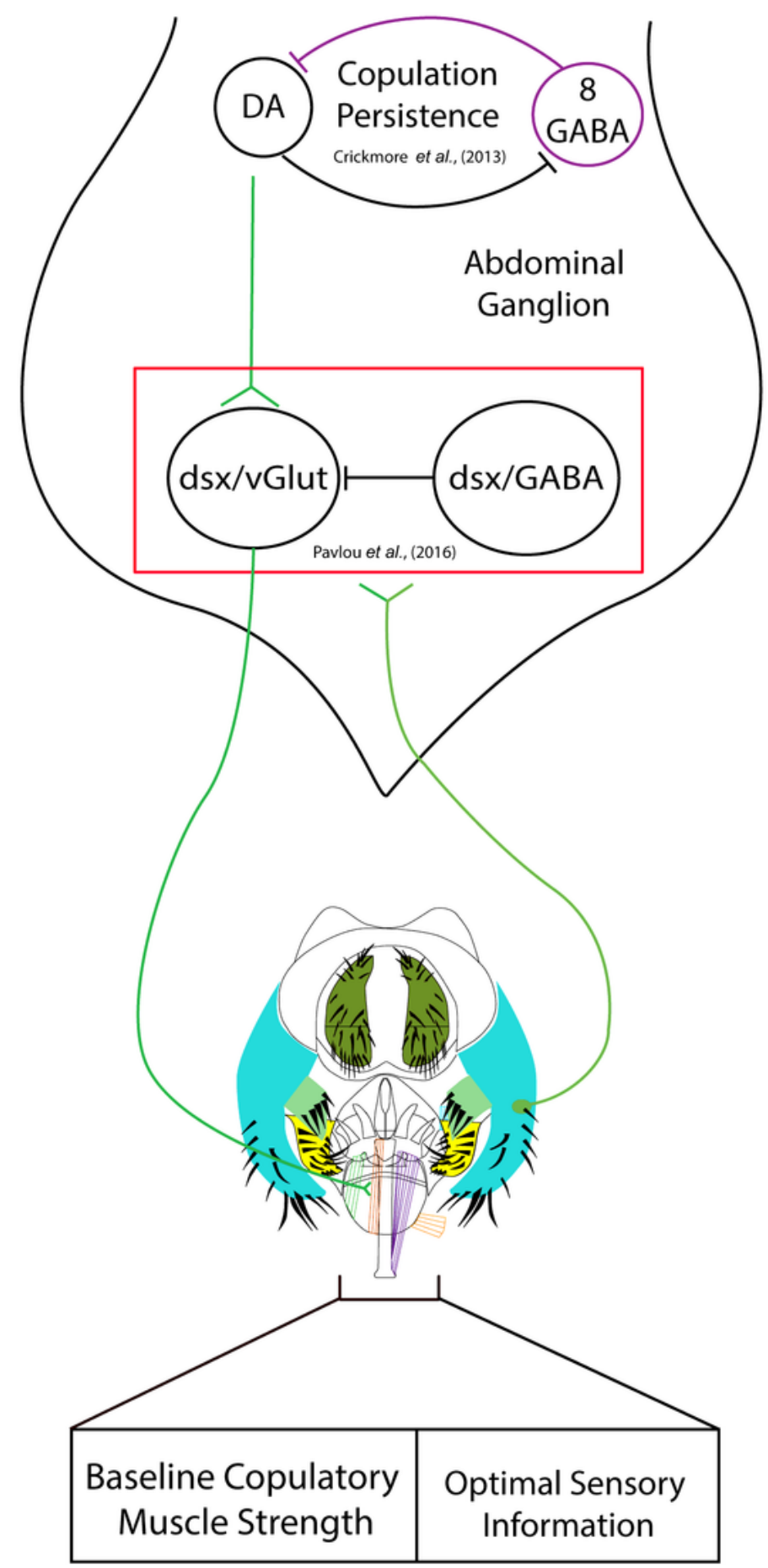

Total tension of copulatory muscles requires for initial copulation persistence before sperm transfer

\section{Figure 5}

Proposed model of how sensory information from the MSNs is incorporated into the neural circuit that governs copulation persistence. Genital coupling requires the activation of the dsx/vGlut motor neurons that innervate the copulatory muscles. The resulting muscular tension is proportional to the activity level of the dsx/vGlut motor neurons, which are antagonistically regulated by dsx/GABA interneurons. These copulation regulating neurons are functionally connected to the axonal terminals of sensory neurons 
from the genitalia bristles34. We propose that a suboptimal sensory input will reduce the total muscular tension due to less activation of the dsx/vGlut motor neurons or more activation of the dsx/GABA interneurons. Copulation persistence ensures the maintenance of copulation before sperm transfer in the presence of stressful stimulus and is regulated by opposing actions of $8 \mathrm{dsx} / \mathrm{GABA}$ and the dopaminergic system in the ventral cord24. This persistence requires optimal sensory input from the genitalia bristles. 